\title{
CORRALLING A DISTANT PLANET WITH EXTREME RESONANT KUIPER BELT OBJECTS
}

\author{
Renu Malhotra, Kathryn Volk, and Xianyu Wang ${ }^{1}$ \\ Lunar and Planetary Laboratory, The University of Arizona, Tucson, AZ 85721, USA; renu@1pl.arizona.edu \\ Received 2016 March 9; revised 2016 May 29; accepted 2016 June 1; published 2016 June 15
}

\begin{abstract}
The four longest period Kuiper Belt objects have orbital periods close to integer ratios with each other. A hypothetical planet with an orbital period of $\sim 17,117$ years and a semimajor axis $\sim 665$ au would have $N / 1$ and $N / 2$ period ratios with these four objects. The orbital geometries and dynamics of resonant orbits constrain the orbital plane, the orbital eccentricity, and the mass of such a planet as well as its current location in its orbital path.
\end{abstract}

Key words: celestial mechanics - Kuiper belt: general - planets and satellites: detection - planets and satellites: dynamical evolution and stability

\section{THE MOST DISTANT KUIPER BELT OBJECTS}

In the outer solar system beyond the orbit of Neptune is the Kuiper Belt, a large belt of minor planets whose dynamics have been understood over the past two decades to be significantly controlled by the gravity of the giant planets Jupiter, Saturn, Uranus, and Neptune, either through secular or resonant perturbations or by gravitational scattering. However, there are a few known Kuiper Belt objects (KBOs) that are unlikely to be significantly perturbed by the known giant planets in their current orbits. In the dynamical classification scheme for the small bodies of the outer solar system, such objects (semimajor axis, $a$, and perihelion distance, $q$, exceeding $150 \mathrm{au}$ and $40 \mathrm{au}$, respectively) would likely belong in a class known as "detached objects," which are distinct from the classical KBOs, the resonant KBOs, and the scattered/scattering KBOs (Gladman et al. 2008). Trujillo \& Sheppard (2014) call them "extreme" KBOs (eKBOs, henceforth). Upon querying the Minor Planet Center's catalog, we identify six KBOs having $a>150$ au and $q>40 \mathrm{au}$; two of these are numbered and four are unnumbered: Sedna (90377), 2010 GB174, 2004 VN112, 2012 VP113, (148209), and 2013 GP136. Their orbital inclinations are in the range 11.9-33.5 (to the ecliptic), not especially remarkable for KBOs. However, all of these objects have extremely large orbital eccentricities, $e \gtrsim 0.7$, suggesting large orbital perturbations in their past histories. This is rather at odds with their current large separations from the known giant planets.

A natural hypothesis is that these objects' orbital dynamics were or are dominated by the gravitational perturbations of a planet in the heliocentric distance range of a few hundred au. Gladman \& Chan (2006) hypothesized a "rogue" planet, a short-lived planet with a mass larger than an Earth-mass, to explain the eKBOs. Trujillo \& Sheppard (2014) proposed an extant hypothetical distant planet to explain an apparent clustering of the arguments of perihelion of the eKBOs. Batygin \& Brown (2016) have also argued for this hypothesis by calling attention to the clustering of the eccentricity vectors and orbit poles of some distant KBOs. These authors suggest a planet mass of $\sim 10 M_{\oplus}$ and an orbit with semimajor axis $a \simeq$ 700 au and eccentricity $e \simeq 0.6$. Fienga et al. (2016) provide an analysis of Cassini tracking data, arguing that for the favored planet parameters of Batygin \& Brown (2016), the residuals in

\footnotetext{
${ }^{1}$ Also: School of Aerospace Engineering, Tsinghua University, Beijing, China.
}

the orbital motion of Saturn are reduced with the existence of such a planet at current true longitude near $120^{\circ}$; however, their analysis cannot exclude a large range of true longitudes $\left(-132^{\circ}, 106^{\circ}\right)$ where the planet would be too distant to sufficiently perturb Saturn.

Here, we report additional support for the extant distant planet hypothesis and narrow the range of its parameters and current location. We point out hitherto unnoticed peculiarities of the orbits of the eKBOs; we find that the orbital period ratios of these objects are close to integer ratios. Such ratios are not dynamically significant unless the eKBOs are in mean motion resonances (MMRs) with a massive planet. We identify an orbital period of the hypothetical planet (HP, henceforth) which is in simple integer ratios with the four most distant eKBOs. We show that the resonant dynamics provide constraints on the planet's orbital parameters and its mass and can also narrow the possible range of its current location in its orbital path.

\section{MEAN MOTION RESONANCES}

The wide range of the perihelion-to-aphelion distances of each eKBO may overlap with those of the HP. In this circumstance, the extreme eccentricities of the eKBOs may be a natural consequence of gravitational scattering encounters with the planet. The dynamical survival of scattered objects over solar system age could be simply due to the long timescales of close encounters at these distances or it could be due to enhanced dynamical stability offered by phase-protection in MMRs with HP. In their analysis, Batygin \& Brown (2016) emphasized the secular effects of the planets in aligning the orbits of the eKBOs, but also noted that transient resonant interactions would be important. Here we investigate the possibility of extant MMRs of the known eKBOs with the unseen planet.

Examining the orbital periods of the six eKBOs as listed in the Minor Planet Center's catalog and ordering them in order of decreasing orbital period (so Sedna is eKBO\#1 with the longest period orbit), we find for the ratios $P_{1} / P_{j}$ :

$$
\text { 1.596, 1.993, 2.666, 3.303, } 6.115 \text {. }
$$

These values are close to the following rational ratios: $8 / 5$, $2 / 1,8 / 3,10 / 3$, and $6 / 1$, which are sufficiently intriguing for further investigation.

We proceeded as follows. We computed the best-fit orbits for these six eKBOs as well as their uncertainties using the 


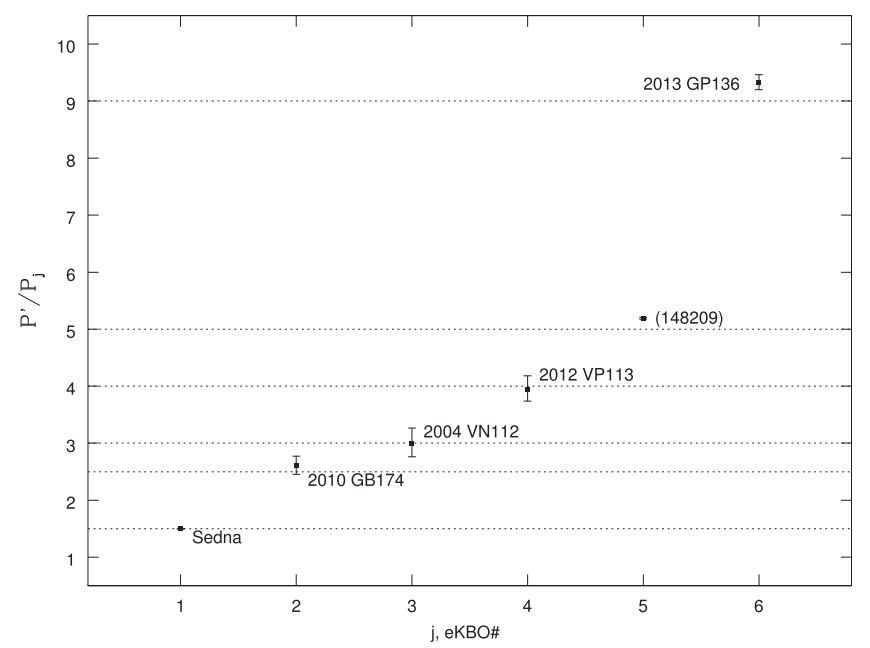

Figure 1. Period ratios of the six eKBOs with the hypothetical planet. The error bars indicate $3 \sigma$ uncertainties. The orbit period $P^{\prime}$ of HP is assumed to be $3 / 2$ times Sedna's best-fit orbital period, $P_{1}=11,411$ years. The dotted horizontal lines indicate the rational ratios $3 / 2,5 / 2,3 / 14 / 1,5 / 1$, and $9 / 1$.

orbit-fitting software developed by Bernstein \& Khushalani (2000); for these orbit fits we included all of the astrometric measurements for the objects listed in the Minor Planet Center. We find the following best-fit semimajor axes and their $1 \sigma$ uncertainties (all quoted in au):

$$
\begin{aligned}
& 506.84 \pm 0.51,350.7 \pm 4.7,319.6 \pm 6.0,265.8 \pm 3.3 \\
& 221.59 \pm 0.16,149.84 \pm 0.47
\end{aligned}
$$

These osculating barycentric elements agree well with the heliocentric MPC orbits when both are converted to heliocentric cartesian positions and velocities.

We then consider some simple possibilities for HP's resonant orbit. Assuming that its orbital period $P^{\prime}$ is longer than $P_{1}$, but not by too much or else its gravitational effects would not be very strong, we consider $P^{\prime} / P_{1}$ values of $2 / 1$ or $3 / 2$ or $4 / 3$. We find that the case of $P^{\prime} / P_{1}=3 / 2$ (hence $a^{\prime} \approx$ $665 \mathrm{au})$ is particularly striking, for it yields period ratios close to $5 / 2,3 / 1$, and $4 / 1$ for $P_{2}, P_{3}$, and $P_{4}$, respectively. The other simple choices $\left(P^{\prime} / P_{1}\right.$ values of $2 / 1$ or $\left.4 / 3\right)$ yield larger integer ratios for $P^{\prime} / P_{2}, P^{\prime} / P_{3}$, and $P^{\prime} / P_{4}$. Although these larger integer ratios cannot be ruled out prima facie, we limit this first study to the small integer ratio MMRs. Other MMRs will be considered in a future study.

In Figure 1 we plot the period ratios $P^{\prime} / P_{j}$ for the best-fit orbits with their uncertainties. We observe that, within $3 \sigma$ uncertainties, four of the six eKBOs have periods near $N / 1$ or $N / 2$ ratios with HP when we take the latter's period to be 1.5 times Sedna's best-fit period. We also observe that the error bars are not insubstantial and one can be skeptical of any perceived coincidence with simple ratios. We remark that our procedure for computing the uncertainties weights all published observations equally and may overestimate the true errors.

Second, one might also be skeptical of the period ratio coincidences because not all possible $N / 1$ and $N / 2$ period ratios in the range shown are occupied. On this point we can remark that (a) the larger $N$ values lead to shorter period orbits in which HP's influence is diminished and Neptune's influence looms larger, potentially destabilizing the resonances with HP, and (b) the small number of currently known eKBOs will occupy only a small fraction of all possible $N / 1, N / 2$ period ratios.

Third, one can ask if the proximity to the resonant ratios is different from random. We answer this question approximately as follows. ${ }^{2}$ For integer values $0<N_{i}<N_{j} \leqslant 10$, there are 31 distinct rational numbers $N_{j} / N_{i}$ in the range $(1,10)$, so the mean distance between said rationals is $\sim 0.29$. We can compare this mean value with the distances of the observed period ratios to the resonant ratios. Taking account of the $3 \sigma$ observational uncertainties, the latter are $0.007,0.27,0.27$, $0.26,0.21$, and 0.46 , respectively (listed in increasing period ratio as in Figure 1). The shortest period eKBO, 2013 GP136, presents the largest distance (from the $9 / 1$ ratio), as is evident in Figure 1. Sedna's very small distance to the $3 / 2$ ratio is explained by its very small orbital uncertainties and because its period ratio of $3 / 2$ is fixed by hypothesis. The remaining four values are similar to the mean distance between the rationals, indicating that they are not strongly distinct from random. However, this statistical argument depends upon how many rationals are allowed and it does not consider any physics of MMRs. We will show later (Section 2.2) that the libration widths of the specific MMRs are similar to these apparent distances from exact resonant ratios; hence, libration in MMRs is possible even with the significant apparent distances from exact resonant ratios. We therefore proceed with investigating the implications of these MMRs for the four longest period eKBOs.

\subsection{Resonant Orbital Geometry Constraints}

Stable resonant orbits of the eKBOs will avoid close approaches with HP and will have a discrete number of geometries for the conjunctions of the eKBO with the planet. These are described by librations of the critical resonant angle

$$
\phi=(p+q) \lambda^{\prime}-p \lambda-q \varpi .
$$

As usual, in the definition of the resonant angle, $p$ and $q$ are integers, $\lambda$ the mean longitudes and $\varpi$ the longitudes of perihelion; we use the prime to refer to orbital parameters of the planet, the parameters of the eKBO are unprimed. Depending upon the orbital eccentricity, $\phi$ may be stable at either 0 or at $\pi$ or at both.

In Figure 2 we illustrate the trace of the resonant orbits (of zero libration amplitude) in the frame co-rotating with the mean angular velocity of HP. Also included here are the traces of a few possible coplanar orbits of HP, with eccentricity $e^{\prime}=0$, 0.17 and 0.4 .

We observe that for Sedna, stable libration is likely only near $\phi=\pi$ and unlikely near $\phi=0$, because the latter would allow small encounter distances. (This is the opposite of the geometry for the low eccentricity regime in which stable (unstable) librations are centered at $\phi=0(\pi)$.) Consequently, conjunctions with HP occur near one of three longitudes, $\pm \pi / 3$ and $\pi$ from Sedna's longitude of perihelion. Moreover, the long term stability of Sedna would limit $e^{\prime} \lesssim 0.41$. Likewise, a coplanar orbit of 2010 GB174 with $\phi=\pi$ limits $e^{\prime}<0.04$; however, in the geometry with $\phi=0$, the limit is $e^{\prime} \lesssim 0.18$. The orbit of 2004 VN112 limits $e^{\prime} \lesssim 0.42$ for $\phi=\pi$.

Of course strict coplanarity of HP is possible for at most one eKBO, because the eKBOs' orbits are not coplanar. Figure 3

\footnotetext{
2 A rigorous statistical analysis finds similar results (S. Mills \& D. Fabrycky 2016, personal communication).
} 


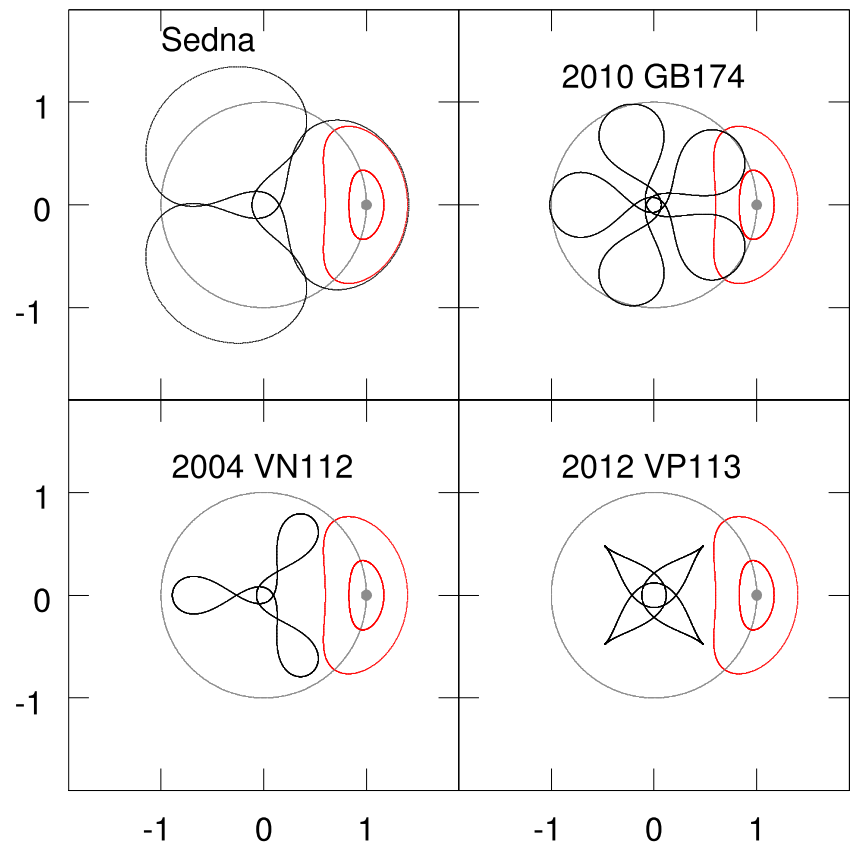

Figure 2. Resonant orbits in the rotating frame are in black. The red loops indicate the trace of HP in possible eccentric orbits of eccentricity 0.17 and 0.4 . Distances are scaled to the semimajor axis of HP. The gray circle is the unit radius.

shows the ecliptic latitude and longitude of the orbit normal vectors of each of the four potentially resonant eKBOs. We see that they are somewhat clustered, approximately within one quadrant of ecliptic longitudes; they have relative inclinations $7^{\circ}-15^{\circ}$ to their mean plane.

The non-coplanarity of the orbits with HP's orbit plane may relax the upper limit on $e^{\prime}$, especially if the eKBO's argument of perihelion $g$ (relative to HP's orbit plane) were near $\pm 90^{\circ}$. This would have the effect of lifting the eKBO's aphelion away from the HP orbit plane and thereby increasing the closest approach distances and enhancing dynamical stability. ${ }^{3}$ Such a configuration is associated with the so-called "periodic orbits of the third kind" envisioned by Henri Poincaré (as noted by Jefferys 1966a) and computed by Jefferys (1966b), Kozai (1969) and Jefferys \& Standish (1972) for the three-dimensional circular restricted three body problem. Such periodic orbits are characterized by simultaneous stationarity of the resonant angle $\phi$ (at 0 or $\pi$ ) and of the argument of perihelion $g$ (at 0 or $\pi$ or $\pm \pi / 2$ ). Physically we can visualize these periodic orbits as having a fixed orbit plane and a fixed eccentricity vector (i.e., the precession rates of both node and pericenter vanish). In the particular case of $g= \pm \pi / 2$, the particle reaches aphelion when it is at its largest excursion away from the plane of the planet.

We computed the values of $g$ relative to the mean plane of the four longest period eKBOs, finding that they are

$$
145^{\circ} .4,-70.4,12^{\circ} .0,-39.3 \text {. }
$$

We look for an alternative HP orbit plane for which $g$ is proximate to $\pm 90^{\circ}$ within some specified tolerance, for all of the four eKBOs. We proceed as follows. Let $\hat{\boldsymbol{n}}$ be the

\footnotetext{
3 This geometry is reminiscent of the dynamics of Pluto's orbit, which librates in an exterior 3/2 MMR with Neptune; Pluto's argument of perihelion is presently $\sim 104^{\circ}$ and it librates about $90^{\circ}$ with a period of about four megayears (Malhotra \& Williams 1997).
}

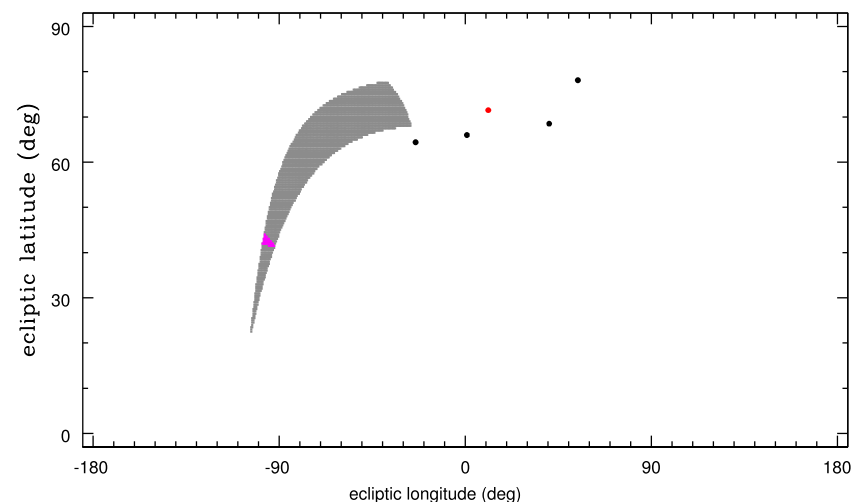

Figure 3. The orbit planes, as described by the ecliptic latitude and longitude of the orbital normal vectors, of the four potentially resonant eKBOs are indicated by the black dots. The red dot indicates their mean plane. The region shaded in gray identifies the planes for which the four eKBOs have $g$ within $45^{\circ}$ of $\pm \pi / 2$. The region shaded in magenta identifies the subset for which the relative inclinations of the eKBOs are also within $10^{\circ}$ of the stationary inclination values at the periodic orbit of the third kind.

(unknown) unit vector normal to the HP orbit plane. Let $\hat{\boldsymbol{c}}_{j}$ be the unit vector in the orbit plane of an $\mathrm{eKBO}$ and normal to its eccentricity vector. We ask whether $\hat{\boldsymbol{c}}_{j}$ can be rotated by no more than a specified amount, $\Delta \omega$, in the eKBO's orbit plane, such that $\hat{\boldsymbol{n}} \cdot \hat{\boldsymbol{c}}_{j}$ changes sign; if so, this would indicate that the eKBO has $g$ within $\Delta \omega$ of $\pm \pi / 2$, near the periodic orbit of the third kind. By demanding that all four eKBOs satisfy this constraint, we identify the range of possible $\hat{\boldsymbol{n}}$. The results of this search are shown in Figure 3, in which we plot in gray the ecliptic longitude and latitude of the allowed $\hat{\boldsymbol{n}}$ 's for $\Delta \omega=45^{\circ}$.

We narrow the possible HP planes further by examining the inclinations of the eKBOs to possible HP orbit planes. Consulting the results of Jefferys \& Standish (1972), we find that the stationary inclinations (relative to HP's orbit plane) are $49^{\circ}, 62^{\circ}, 54^{\circ}$, and $57^{\circ}$, respectively, for the specific MMRs and the observed eccentricities of the four eKBOs. Of course, these eKBOs are likely librating with non-zero amplitude about the pertinent periodic orbit, so we look for those $\hat{\boldsymbol{n}}$ 's that yield inclinations within $10^{\circ}$ of these values. Carrying out this search, we find that the range indicated in magenta in Figure 3 satisfies this constraint. We conclude that an HP orbit plane with inclination near $i \approx 48^{\circ}$ and a longitude of ascending node near $\Omega \approx-5^{\circ}$ allows all four resonant eKBOs to be in proximity of the periodic orbits of the third kind.

\subsection{Planet Mass}

Resonant perturbations from HP provide phase-protection if an eKBO is sufficiently close to the exact resonant orbit. How close is close enough depends upon the planet mass. We now quantify this condition.

In the usual canonical variables,

$$
\begin{gathered}
J_{1}=L=\sqrt{G M_{\odot} a}, \quad \theta_{1}=l \text { (mean anomaly) } \\
J_{2}=G=L \sqrt{1-e^{2}} \\
\theta_{2}=g(\operatorname{argument} \text { of pericenter }) \\
J_{3}=H=G \cos i \\
\theta_{3}=h \text { (longitude of ascending node), }
\end{gathered}
$$


the orbital perturbation equations are given by

$$
\dot{J}_{i}=-\frac{\partial \mathcal{H}_{\text {pert }}}{\partial \theta_{i}}, \quad \dot{\theta}_{i}=\frac{\partial \mathcal{H}_{\text {pert }}}{\partial J_{i}},
$$

where the perturbation Hamiltonian is

$$
\begin{aligned}
\mathcal{H}_{\text {pert }} & =-\mathrm{Gm}^{\prime}\left(\frac{1}{\left|\boldsymbol{r}-\boldsymbol{r}^{\prime}\right|}-\frac{\boldsymbol{r} \cdot \boldsymbol{r}^{\prime}}{r^{\prime 3}}\right) \\
& \simeq-\frac{\mathrm{Gm}^{\prime}}{a^{\prime}} \mathcal{A}(L, G, H) \mathcal{C}(\phi) .
\end{aligned}
$$

Here, the first equation gives the Newtonian perturbation potential $\left(\boldsymbol{r}, \boldsymbol{r}^{\prime}\right.$ are the position vectors of an $\mathrm{eKBO}$ and of HP, respectively, in Jacobi coordinates (e.g., Brouwer \& Clemence 1961); i.e., they are relative to the barycenter of the Sun and the known planets), whereas the second equation gives an approximation for the resonant perturbation. The dependence of the resonant perturbation on the canonical variables can be expressed as a product of an amplitude $\mathcal{A}(L, G, H)$, which is a function of only the canonical momenta, and a phase $\mathcal{C}(\phi)$ which is an even function of the resonant angle. ( $\mathcal{A}$ also depends on the orbital parameters of HP, which will be assumed to be constant.) Without loss of generality, we can define $\mathcal{A}$ and $\mathcal{C}$ such that $|\mathcal{C}(\phi)|<1$ for $0 \leqslant \phi<2 \pi$. For the eKBOs whose extreme eccentricities are near unity, the magnitude of $\mathcal{A}$ will be on the order of a few. When we require numerical values (below), we will adopt $\mathcal{A}=3$; but this value can, in principle, be determined more accurately with numerical averaging of the perturbation potential.

The mean motion of the eKBO is $n=\left(G M_{\odot}\right)^{2} / L^{3}$. Therefore, the resonant perturbations of the mean motion are given by $\dot{n}=-(3 p n / L) \partial \mathcal{H}_{\text {pert }} / \partial \phi$, whence we obtain the following equation of motion for $\phi$ :

$$
\ddot{\phi} \simeq-\frac{3 p^{2} m^{\prime} a}{M_{\odot} a^{\prime}} n^{2} \mathcal{A} \mathcal{S}(\phi),
$$

where $\mathcal{S}(\phi)=-d \mathcal{C}(\phi) / d \phi$. This equation for $\phi$ resembles the nonlinear pendulum. The small amplitude oscillation frequency is given by

$$
\omega_{0} \simeq\left|\frac{3 p^{2} m^{\prime} a}{M_{\odot} a^{\prime}} \mathcal{A}\right|^{\frac{1}{2}} n
$$

For the resonances of interest, the corresponding libration periods are on the order of a few megayears for a few-Earthmass planet.

By analogy with the pendulum, we divine that $\phi$ (hence the conjunction longitudes $\lambda_{\mathrm{c}}$ ) can execute librations about stable equilibrium points provided $|\dot{\phi}|=|p \delta n+q \dot{\varpi}| \lesssim 2 \omega_{0}$. Thus, the required condition for resonance is that the deviation of the mean motion of the eKBO from the exact resonant value, $(p+q) n^{\prime} / p$, should not exceed $\sim 2 \omega_{0} / p$. Then, by Kepler's third law, the resonance width in semimajor axis is given by

$$
\Delta a_{\mathrm{res}} \simeq a\left|\frac{16 m^{\prime} a \mathcal{A}}{3 M_{\odot} a^{\prime}}\right|^{\frac{1}{2}} \simeq 0.007 a\left|\frac{m^{\prime} a \mathcal{A}}{3 M_{\oplus} a^{\prime}}\right|^{\frac{1}{2}} .
$$

Computing the widths of the $3 / 2,5 / 2,3 / 1$, and $4 / 1$ resonances for the four eKBOs of interest, we find that a
$10 M_{\oplus}$ planet yields $\Delta a_{\text {res }}$ values comparable to the uncertainties in the semimajor axes of these objects.

The more severe condition on the planet mass obtains when we demand that the eKBOs are in libration about periodic orbits of the third kind. The frequency of small amplitude oscillations about these periodic orbits ${ }^{4}$ is given by

$$
\omega_{0} \approx c n \frac{m^{\prime} a^{3}}{M_{\odot} b^{\prime 3}} e \sin i
$$

where $n, a, e, i$ are a resonant eKBO's mean motion, semimajor axis, eccentricity, and inclination (relative to HP's orbit plane), $b^{\prime}=a^{\prime} \sqrt{1-e^{\prime 2}}$ is the planet's semi-minor axis, and $c$ is a numerical coefficient whose asymptotic value is $\sim 5.8$ for $a \ll$ $a^{\prime}$ ( $c$ will be larger for $a$ approaching $a^{\prime}$ ). For numerical estimates we adopt $e^{\prime}=0.2$ and $c=8$. We find that for $m^{\prime}=10 M_{\oplus}$, the small amplitude oscillation periods for the four eKBOs are 0.6-0.94 Gyr, a factor of a few smaller than the age of the solar system. We conclude that $10 M_{\oplus}$ is approximately the minimum mass necessary to maintain periodic orbits of the third kind for the four eKBOs.

\subsection{Planet's Current Location in Its Orbit}

Each resonant eKBO constrains the longitude of HP because the critical resonant angle must librate about either $0^{\circ}$ or $180^{\circ}$. For illustration, consider the libration of $\phi=3 \lambda^{\prime}-2 \lambda-\varpi$ about $180^{\circ}$ for Sedna's $3 / 2$ resonant orbit. Given that Sedna is presently near its perihelion, $\lambda \approx \varpi$, the planet can be in proximity to one of three longitudes: $60^{\circ}$, $180^{\circ}$, or $300^{\circ}$ from Sedna's longitude of perihelion, $\varpi$. The trace of the resonant orbit in the rotating frame (Figure 2) shows that libration of $\phi$ would allow the planet to be located as far as $\sim 54^{\circ}$ from these longitudes.

This means that only three small zones of $\pm 6^{\circ}$ around each of three locations, $\varpi, \varpi+120^{\circ}$ and $\varpi-120^{\circ}$ are excluded for the planet's present location. For $e^{\prime}=0.2$, we find that the excluded zone is a little larger, \pm 7.5 around each of the same three locations.

However, such geometric estimates of exclusion zones do not account for the planet's gravity. To account for this, we computed surfaces of section for the circular planar restricted three body problem for several values of the planet mass in the range of $10^{-6}-10^{-3} M_{\odot}$, for the neighborhood of the $3 / 2,5 / 2$, $3 / 1$, and 4/1 MMRs and the extreme eccentricities of the four eKBOs $(0.85,0.87,0.85$ and 0.7 , respectively). Figure 4 shows a sample of these for planet mass $3 \times 10^{-5} M_{\odot}$ (approximately $\left.10 M_{\oplus}\right)$. From these surfaces of section, we determine the range of excluded longitudes of the planet by measuring the complement of the large libration zones of the $3 / 2$ and 5/2 MMRs. We also conservatively exclude a small range, $\pm 5^{\circ}$, around the homoclinic points of the $3 / 1$ and $4 / 1$ resonant eKBOs. Figure 5 shows our estimates of the excluded zones, projected on the sky, for a planet of mass $3 \times 10^{-5} M_{\odot}$; we plot a representative case of the planet's low inclination orbit (in green) and also a representative case of its high inclination orbit (in red). (Note that a range of orbital planes near each of these representative cases is allowed by our

\footnotetext{
4 These periodic orbits, embedded in the $N / 1$ or $N / 2$ MMRs, are often inaccurately referred to as "Kozai within MMR." They can be considered to be the discrete sequence whose limit for $N \gg 1$ is the Kozai-Lidov resonance (Kozai 1962; Lidov 1962). Equation (10) is obtained in this limit.
} 

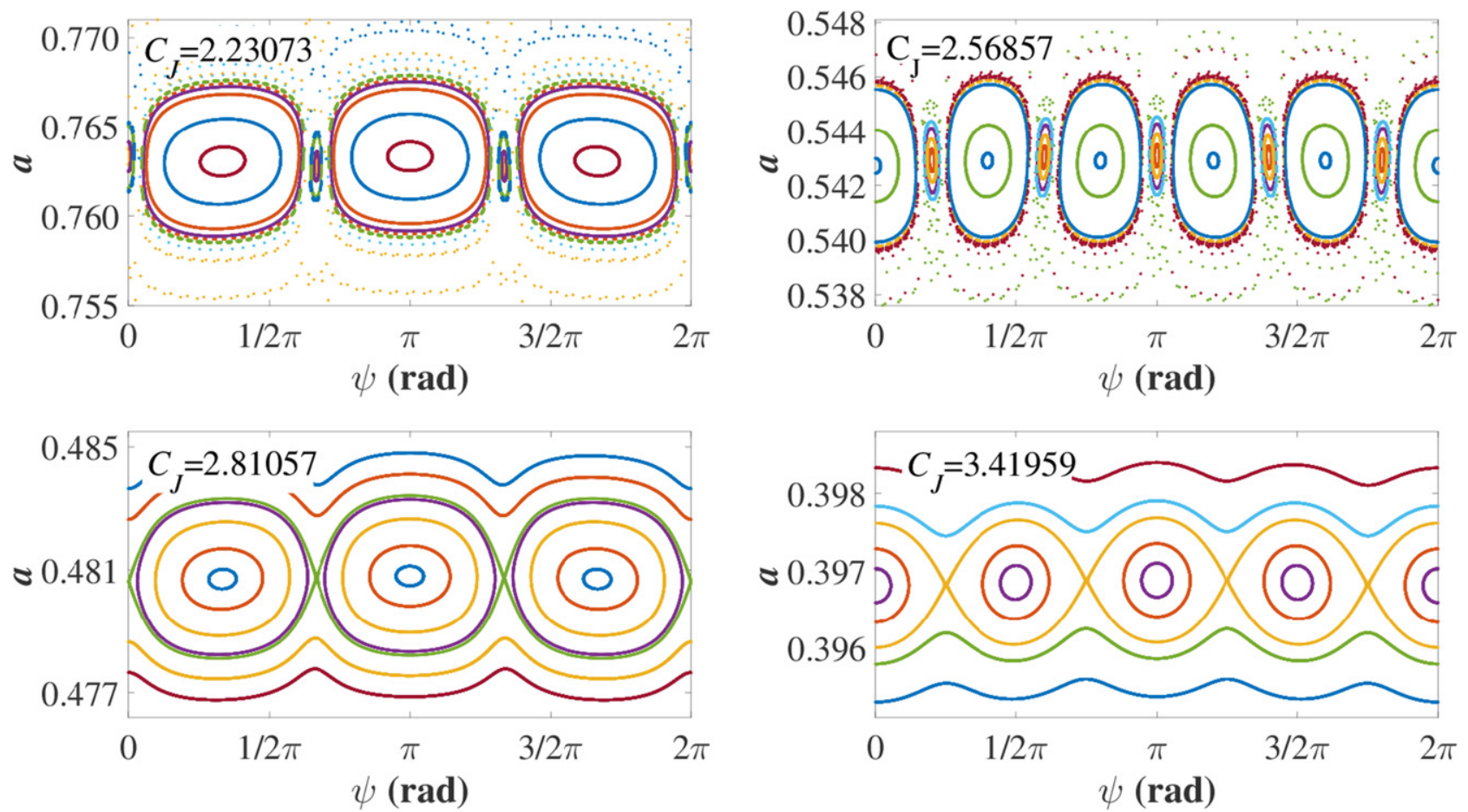

Figure 4. Surfaces of section for the circular planar restricted three body problem, for mass ratio $m_{2} /\left(m_{1}+m_{2}\right)=3 \times 10^{-5}$, and Jacobi constant values as indicated. These are phase space portraits in the vicinity of the $3 / 2,5 / 2,3 / 1$, and $4 / 1$ resonant orbits of eccentricity $0.85,0.87,0.85$ and 0.7 , respectively. The abscissa, $\psi$, measures the angle between the planet and the particle's pericenter; the ordinate is the particle's barycentric semimajor axis, in units of the circular orbit radius of the planet.

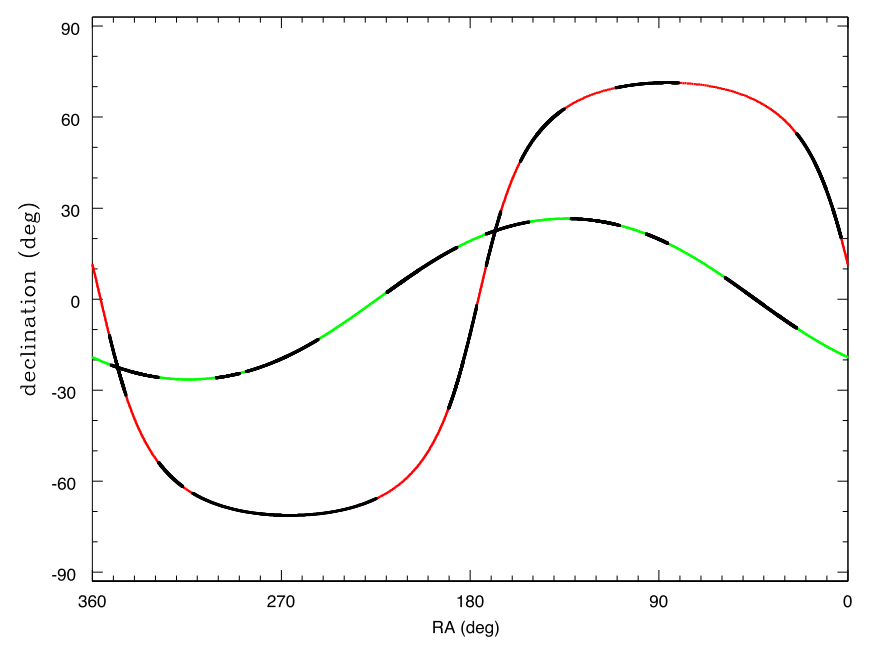

Figure 5. Possible locations of HP in the sky: in green for a representative low inclination orbit and in red for a representative high inclination orbit; the zones in black are excluded by the resonant eKBOs. A range of at least several degrees near each of these representative planes is allowed by our calculations.

calculations.) We find that approximately half of the ecliptic longitudes are excluded in both cases; the excluded zone is not contiguous.

\section{SUMMARY AND DISCUSSION}

We noticed that the four known longest period KBOs (Sedna (90377), 2010 GB174, 2004 VN112, and 2012 VP113) could all be in MMRs with an unseen distant planet. The set of resonances is not uniquely determined. In the present work, we investigated the case of a HP of orbit period $P^{\prime} \simeq 17,117$ years (semimajor axis $a^{\prime} \simeq 665 \mathrm{au}$ ), which would have dynamically significant $N / 1$ and $N / 2$ period ratios with these four eKBOs. We give a broad sketch of how resonant geometries and resonant dynamics of these high eccentricity eKBOs help narrow the range of planet parameters; many details in this analysis would benefit from deeper investigation in future work and potentially improve planet parameters further.

Our calculations suggest two possibilities for the planet's orbit plane: a plane moderately close to the ecliptic and near the mean plane of the four eKBOs $\left(i \simeq 18^{\circ}, \Omega \simeq 101^{\circ}\right)$, or an inclined plane near $i \simeq 48^{\circ}, \Omega \simeq-5^{\circ}$. The former offers dynamical stability of the four eKBOs by means of libration of the critical resonant angles and the latter offers enhanced dynamical stability by means of additional libration of the argument of perihelion associated with periodic orbits of the third kind (a class of periodic orbits of the three-dimensional restricted three body problem).

In the case of coplanar MMRs, the planet's orbital eccentricity is limited to $e^{\prime} \lesssim 0.4$ by the putative $3 / 2$ resonant orbit of Sedna, and to $e^{\prime} \lesssim 0.18$ by the putative $5 / 2$ resonant orbit of 2010 GB174. For non-coplanar orbits, including the periodic orbits of the third kind, the planet's eccentricity may be larger than these limits. Preliminary numerical analysis finds that for relative inclinations up to $15^{\circ}$, stable resonant librations are possible but uncommon for $e^{\prime} \gtrsim 0.3$.

A planet mass of $\sim 10 M_{\oplus}$ defines resonance widths similar to or exceeding the semimajor axis uncertainties of the observed eKBOs. Resonant libration periods would be on the order of a few megayears, much shorter than the age of the solar system. In the case of the inclined planet orbit, a planet of $\gtrsim 10 M_{\oplus}$ also supports librations about the periodic orbits of the third kind, with libration periods shorter than the age of the solar system. Both these estimates support the conjecture that the period ratio coincidences are of dynamical significance. 
We determined exclusion zones of the current location of the planet in its orbital path (Figure 5) based on the MMRs of all four eKBOs; these exclude just over half of the orbital path, assuming a $10 M_{\oplus}$ planet.

Our analysis supports the distant planet hypothesis, but should not be considered definitive proof of its existence. The orbital period ratios have significant uncertainties, so the nearcoincidences with MMRs may simply be by chance for a small number of bodies. We encourage further observations of these eKBOs to reduce the uncertainties in their orbital periods; a factor of $\sim 3$ reduction in the uncertainties would provide a clear test of our specific MMR hypothesis for a $\sim 10 M_{\oplus}$ planet. Future work should also examine other possible sets of MMRs.

As a final point, we note that the long orbital timescales in this region of the outer solar system may allow formally unstable orbits to persist for very long times, possibly even to the age of the solar system depending on the planet mass; if so, this would weaken the argument for a resonant planet orbit. In future work, it would be useful to investigate scattering efficiency as a function of the planet mass, as well as dynamical lifetimes of non-resonant planet-crossing orbits in this region of the outer system. Nevertheless, the possibility that resonant orbital relations could be a useful aid to prediction and discovery of additional high mass planets in the distant solar system makes a stimulating case for renewed study of aspects of solar system dynamics, such as resonant dynamics in the high eccentricity regime, which have hitherto garnered insufficient attention.
R.M. thanks Scott Tremaine, Daniel Fabrycky, Sean Mills, and Anthony Dobrovolskis for helpful comments on this work; Eric Christensen for an observer's perspective; and David Frenkel for many clarifying discussions. R.M. and K.V. acknowledge funding from NASA (grant NNX14AG93G). X.W. acknowledges funding from National Basic Research Program of China (973 Program) (2012CB720000) and China Scholarship Council. This research made use of the NASA Astrophysics Data System Bibliographic Services.

\section{REFERENCES}

Batygin, K., \& Brown, M. E. 2016, AJ, 151, 22

Bernstein, G., \& Khushalani, B. 2000, AJ, 120, 3323

Brouwer, D., \& Clemence, G. M. 1961, Methods of Celestial Mechanics (New York: Academic)

Fienga, A., Laskar, J., Manche, H., \& Gastineau, M. 2016, A\&A, 587, L8 Gladman, B., \& Chan, C. 2006, ApJL, 643, L135

Gladman, B., Marsden, B. G., \& Vanlaerhoven, C. 2008, in Nomenclature in the Outer Solar System, ed. M. A. Barucci et al. (Tucson, AZ: Univ. Arizona Press), 43

Jefferys, W. H. 1966a, AJ, 71, 99

Jefferys, W. H. 1966b, AJ, 71, 566

Jefferys, W. H., \& Standish, E. M., Jr. 1972, AJ, 77, 394

Kozai, Y. 1962, AJ, 67, 591

Kozai, Y. 1969, PASJ, 21, 267

Lidov, M. L. 1962, P\&SS, 9, 719

Malhotra, R., \& Williams, J. G. 1997, in Pluto's Heliocentric Orbit, ed. S. A. Stern, \& D. J. Tholen (Tucson, AZ: Univ. Arizona Press), 127

Trujillo, C. A., \& Sheppard, S. S. 2014, Natur, 507, 471 